\title{
CAPACITY OF SPACES OF PROPERTIES Formulae, Approximations and Qualitative shapes
}

\author{
Jiri Bila \\ Czech Technical University in Prague \\ Institute of Instrumentation and Control Engineering \\ Technicka 4, 16607 Prague 6 \\ Czech Republic \\ bila@vc.cvut.cz
}

\begin{abstract}
This article focuses on the exploration of spaces and models in which we describe the behavior of complex systems as special shapes. We understand these shapes both as a configuration of properties and their values, and on the other, as the formation of symbols and their manifestations. The article discusses three basic types of shapes: formulae, approximations and qualitative shapes. Their analysis then arrives at the capacities of the spaces to display these shapes. The central tool of analysis is the combination of Matroid Theory and Ramsey theory of graph.

By systematical analysis of formulae we get the concepts of additional variables and their number. We use basic relations based on the terms matroid, its base, and Ramsey numbers. These relations are then generalized to the field of approximations and qualitative shapes. The article points to the possibilities of expanding the spaces of properties including those that are not available by measurement but are detectable as emergences.
\end{abstract}

Keywords: Structural invariant, matroid, matroid bases, Ramsey theory of graph, chaos, fractal dimension

\section{Introduction}

A great part of a practical mathematics is covered by formulae. Formulae express on one side relations between variables and constants on the other side the method and the description of their application. Formulae may be understood as codes of some shapes. Sometimes we are not sure what they really code. For example formulae for DNA could code not final shape but the process that resulted in this shape [2]. If it was truth we have lost a substantial part of the research. Formulae are interpreted in various environments. They are tuned and verified. Here we can rely on human thoroughness, which does not allow the formula that generates great errors to survive. Consider, for example, the formula for calculating lift on the wing of the aircraft. (With what worries we might have been travelling on holiday.)

In this sense, we passed a long way through the development of the gallery of formulae and we will probably not try to refute some of them. Not even in this article. However, in some cases, we suspect that the formula does not express everything about the system under review. If we do not get a formulae describing the behavior of the system for a variety of reasons (this may be the case for a description of ecosystem behavior or behavior of the cardio-vascular system of a living organism), we try to develop so-called approximations. It may be because we do not know the relationships between variables or we do not know all the variables. It can also be because we have only data about behavior, not relationships that allow us to describe and explain the function of the system. In this paper we will describe approximations that reduce algorithms and computational processes in the field of quantum chemistry, e.g., [22], or approximations of dynamic systems by certain integral properties, such as complexity, irregularity, and they are expressed by special functions such as capacitive, correlation and fractal dimension or by Lyapunov exponent. This article also touches on so-called qualitative shapes that continue beyond the area of approximations.

A few words to organization of the paper: Section 2 contains a short list of related works. In Section 3 there are introduced essential conceptual and technical background for the proposed method. Sections 4, 5, and 6 contain application of the proposed method in fields "Shapes formulae", "Shapes approximations" and "Qualitative shapes".

\section{Related Works}

Although the inspirer of this article, I. Stewart [2], dreams about new mathematics suited to working with shapes as complexes advances in this area are not quite pervasive. 
As a kernel for new mathematics, Stewart has named dynamic systems, chaos, and violence of symmetries, fractals, and cellular automata. We deal with the two in this article (Dynamic Systems and Chaos) and violence of symmetries we discussed at work [4]. We also agree on the goals of our efforts. I quote his statement, "Shapes are emerging phenomena of nature." or, "We will never understand how sand dunes occur in the desert, if we "push" these phenomena into rigid mathematical structures." However, if we bind our central concept of "additional and hidden variables," Stewart is over. Hidden variables, especially thanks to the Copenhagen School of Quantum Mechanics, are not welcome. (There is one concept of quantum mechanics, published by De Brolie [16] which, as a hidden parameter, provides a soft rippling membrane. It carries out all the essential phenomena in the structures of the atoms and as a result he gets a deterministic variant of the quantum mechanics where each electron has its calculated speed and coordinates.) With relatively high attention we have dealt with the interpretation of our theory in the field of formulae and approximations in the field of complex systems in material sciences and in the research of the properties of special crystals to which our research has been oriented in recent years [6], [7], [8], [9], [21], [20], [22].

With the topic "Capacity of Spaces of Properties" are associated some problems from the area of deterministic chaos. There are large reserves here, especially in works [10], [17] and [19]. From our sources we will recall works on chaos in Heart Rate Variability [11], [23] and in meteorological data [12]. The topic "Capacity of Spaces of properties" is related to works dealing with complexity [1]. Sometimes the authors are still astonished by "shapes nature" in the Universe and they are looking for the laws of how these shapes were created.

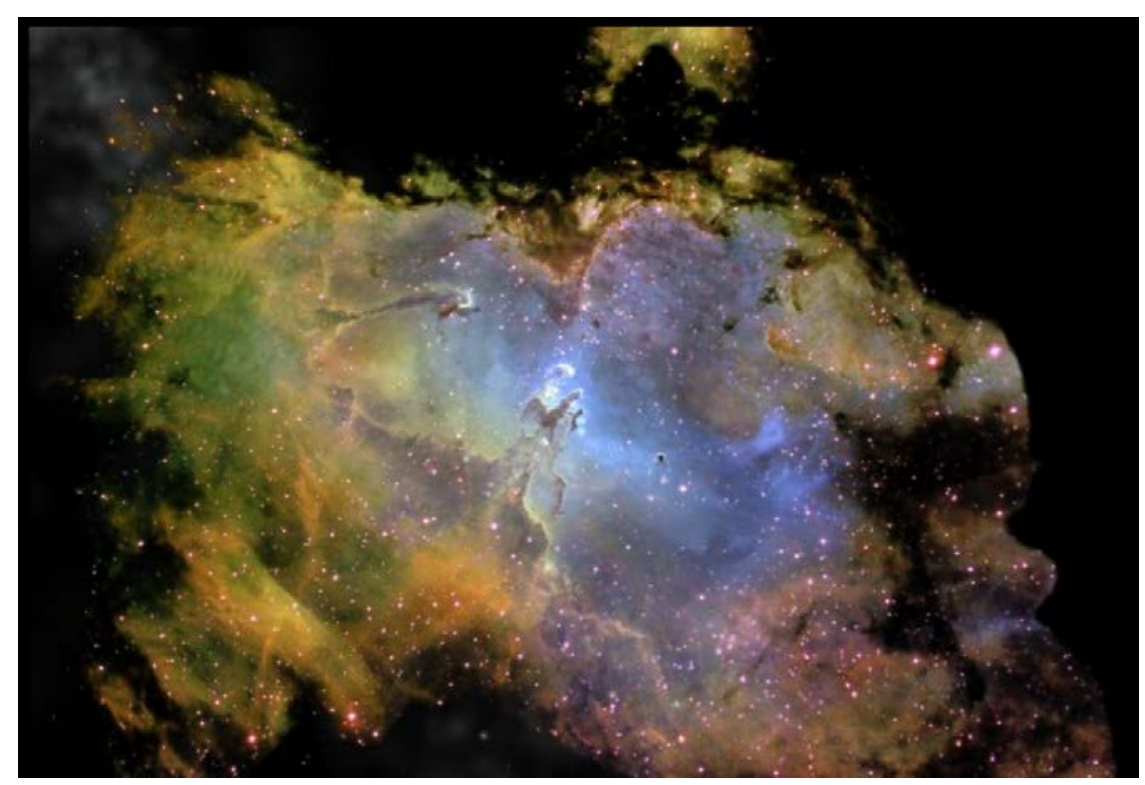

Figure 1: The Nebula in the Eagle

Fig. 1 is given to support our view that differential analysis or formal grammar is not the right way. We quote here also a desire of Stewart [2] to work with shapes, like the whole. Not with their fragments, parsed according to some variables and spaces. Figure 1. also serves as an inspiration to the area of qualitative shapes that we have touched in resources [5], [18] and [21].

\section{Preliminaries}

In technical part of our paper we will interconnect two fields: Theory of Matroids and [13] and Ramsey Theory of Graph [14]. We have used this synthesis as a methodological route many times, e.g., in [4] [5] [6] and it is still interesting how strong and surprising it is. A few sentences about our approach:

Matroid base generates dependent elements. Extensions of some matroid base are possible only from the outside. It is a phenomenon that leads to an increase in the dimension of the original matroid. This can mean both physical shift and knowledge progress. (Extension of some matroid basis by a new independent variable is a way for improving the function of formulae.)

Discovering independent sets and bases on a matroid associates the discovery of perfect subgraphs on a perfect chart.

Let's look at some basic concepts now in more detail. Matroid has the following pleasant properties [13]:

- It is possible to construct it for each set of elements (carrier of the system) when we have the relation of independence or when they are given independent sets. 
- If the relation of independence exists it is easy (in most cases) to associate it with a semantic content (according to real conditions).

- Matroid is usually introduced [12] as the following structure

$$
M=\left\langle X, \mathbf{I N D},\left\{N_{1}, N_{2}, \ldots, N_{n}\right\}\right\rangle=\langle X, \boldsymbol{M B}\rangle,
$$

where $X$ is the ground set of elements (components), IND is a relation of independence, $N_{1}, N_{2}, \ldots, N_{n}$ are independent sets and $\boldsymbol{M B}$ is a set of matroid bases. Matroid bases are maximum (according to cardinality) independent sets. With advantage is used in practical cases relation Dependence (DNT).

One of definitions of Dependence (DNT) relation that respects a problem factor $\alpha$ is the following one:

Definition (1). Elements $x_{1}, x_{2} \in X$ are Dependent (DNT $\left.\left(x_{1}, x_{2} \mid \alpha\right)\right)$ with regard to a given problem factor $\alpha$ if one (or two, three or four) of the following conditions hold(s):

(i) Elements $x_{1}, x_{2}$ contribute to a given problem factor $\alpha$ in the same way or in similar ways, with the same or similar means and principles, taking into account elements from $X$. (Expert criterion.)

(ii) There are changes (variations) of $x_{1}$ which are associated with changes (variations) of $x_{2}$ (or vv.) - with regard a given problem factor $\alpha$ and with regard to elements from $X$.

(iii) The application of $x_{1}$ implies the application of $x_{2}$ (or vv.) - with regard a given problem factor $\alpha$ and with regard to elements from $X$.

(iv) The application of $x_{1}$ excludes the application of $x_{2}$ (or vv.) - with regard a given problem factor $\alpha$ and with regard to elements from $X$.

For our conditions holds simple theorem:

Theorem (1).

$$
\forall x_{1}, x_{2} \in X,\left(\operatorname{Not}\left(\mathbf{D N T}\left(x_{1}, x_{2} \mid \alpha\right)\right)\right) \Leftrightarrow\left(\mathbf{I N D}\left(x_{1}, x_{2} \mid \alpha\right)\right) .
$$

In case that relation IND is considered as a binary relation and it is possible to use the following consequences:

The bases $(\boldsymbol{M} \boldsymbol{B})$ will be constructed as perfect sub-graphs in a perfect graph (each node is connected with all other nodes of $X$ ). Edges of this graph $G_{p}$ on $X$ represent a common symmetric relation (at least equivalence) defining concept "compartment".

The independent and dependent elements in the perfect graph are easy constructed by coloring the edges of this complete graph by two colors and the formalism of Ramsey numbers $-\mathrm{R}(\# B, \# Y), B \in \boldsymbol{M} \boldsymbol{B}$ is offered to be used. We introduce now a free formulation of relations on a perfect graph $G_{p}$ colored by two colors as a consequence of famous Ramsey theorem [14, 15]:

Theorem (2). A perfect graph $G_{p}=(V, E)$ with $n$ nodes where each edge belongs to class $A$ or to class $B$ contains a complete subgraph with a nodes connected by edges from class $A$ or a complete subgraph with $b$ nodes connected by edges from class $B$.

Such a number $\mathrm{R}(a, b)=n$ is called Ramsey number.

\section{Shapes, Formulae and Equations}

It's not always clear, but the left side of the formula usually represents the dependent element that is calculated from the right side elements. In other words, the entire matroid has the number of elements contained in the formula (but may have more). The part of it is a part of base.

However, because we do not know how many elements actually are in the base, we will work with the numbers we know from the formulae with respect that the number of other (additional) variables could be higher than is deduced from \#B (i.e., more than \#X).

Furthermore, we continuously use Ramsey numbers to estimate the number of elements of the base and the matroid. We will use the scheme: Considering the relation (1) then the number of variables in the formula should be equal to the number of matroid elements $\# X=\mathrm{R}(3, \# B)$ where $\# B$ denotes the cardinality (number of elements) of the base. It is obvious that many formulae contain only some variables from the matroid.

Example: If the formula has 5 variables and the base has 4 elements then $\mathrm{R}(3,4)=9$. The lowest number of matroid elements is 9 - there are potentially 4 other (additional) variables.

Variables, in most cases, represent certain characteristics of the system we are investigating. We usually attribute to them some interpretable content and meaning. So if we come to the possibility of other features, it unusually expands our potential cognitive possibilities in learning the system.

Note (1). We work with the lowest possible Ramsey numbers where the first number is always 3. 
Note (2). Other (additional) variables are always dependent on the base (even if they are unknown). Therefore, the additional variables are not hidden variables, and practically do not have to affect the success of the formula. On the other hand, they may represent some simplification or modification of the system.

For Ramsey's numbers, (some of which are listed in [15]), some derived truths hold, e.g., R( $m, 2)=m$. This is interesting for the following fact:

A large number of elementary laws of physics have 3 elemental matroids, $\mathrm{R}(3,2)=3$, i.e., they have twoelement bases.

$$
\begin{aligned}
& F=m \cdot a \quad \ldots \text { Newton's law of power, } \\
& J=m \cdot r^{2} \quad \ldots \text { moment of inertia, } \\
& E=1 / 2 m \cdot v^{2} \ldots \text { kinetic energy, } \\
& U=R \cdot I \quad \ldots \text { Ohm's law of tension, } \\
& \Phi=B \cdot S \quad \ldots \text { Magnetic induction flow, } \\
& Q=C \cdot U \quad \ldots \text { Coulomb's law on electric charge, } \\
& E=F \cdot Q \quad \ldots \text { Intensity of electric field, } \\
& \text { etc. }
\end{aligned}
$$

These equations do not induce any further (additional) variable and they are from our point of view complete. Further analysis of basic physical laws leads to the discovery of possible (additional) variables, e.g., the JouleLenz Law of Joule Heat:

$$
Q_{J}=R \cdot I^{2} \cdot t
$$

where $Q_{J}$ is Joule Heat, $R$ is an ohm resistance of the ohm resistance of the conductor, $I$ is the current passing through the conductor, and $t$ is the time the current passes through the resistance. The result is the heat in Joules that is thus obtained.

Our analysis: We have 4 variables, of which 3 are bases $\mathrm{R}(3, \# B)=\mathrm{R}(3,3)=6$ - two possible other variables. Thus, the process of generating heat by flow of electric current through the conductor could be explained with two further features.

However, there are cases are much more complicated. (We introduce here examples from the field of "complex systems in material sciences" and in the research of properties of special crystals in recent years, [6, 7, 8]). Debey temperature [7]: Debey temperature $\left(\Theta_{D}\right)$ is associated with upper limit of the phonon frequency in a crystal lattice and can be calculated according to following equation:

$$
\Theta_{D}=(h / k)\left[3 n / 4 \pi\left(\rho \cdot N_{A} / m_{M U}\right)\right]^{1 / 3} v_{m},
$$

where $h$ and $k$ are Plank and Boltzmann constants, $n$ is the number of atom per formula unit, $N_{A}$ is Avogadro's number, $\rho$ is the density of compound, $m_{M U}$ is a molecular mass per formula unit, $v_{m}$ is an average wave velocity.

Our analysis: matroid $M=\left(\Theta_{D}, n, \rho, m_{M U}, v_{m}\right)$ has 5 variables and 4 of them represent the basis. Capacity of variables is 9 . We consider about 4 other (additional) variables ( 4 further properties). Here we introduce only 2 of them: longitudinal elastic wave velocity $v_{l}$ and transverse elastic wave velocity $v_{t}$. Both they are functions of $\rho$.

Total energy [8]: I order to investigate the ground state properties of NaAuS system, the total energy is calculated using GGA (General Gradient Approximation) - PBE (Perdew Becke Ernzerhof) scheme. The calculated total energies are fitted to Murnaghans equation of the state:

$$
E(\text { tot })=B_{0} \cdot V / B_{0 p}\left[\left(\left(V_{0} / V\right)^{B_{0 p}} / B_{0 p}-1\right)+1\right]+E_{0}
$$

where $E_{0}$ is the ground state energy, $V$ is the actual volume of the cell and, $V_{0}$ is ground state volume, $B_{0}$ is bulk modulus and $B_{0 p}$ is its derivation according to pressure.

Our analysis: The formula has 6 variables $M=\left(E\right.$ (tot), $\left.B_{0}, B_{0 p}, V_{0}, V, E_{0}\right)$ from which 5 variables belongs to basis. $\mathrm{R}(3,5)=14$, it means 8 further (additional) variables.

Here's another case related to article [6]. To calculate the electron structure of solids, one of the most accurate methods is the Full Potential Linearized Augmented Plane Wave (FPLAPW) method. (This was also used to calculate the atomic coordinates of the compound $\mathrm{C} 13 \mathrm{H} 10 \mathrm{O} 4 \mathrm{~S}$, which we explored in [6]). This is a combination of the Full-Potential (LAPW) method of linearized associated plane waves. This method solves the Kohn-Sham equation (special modification of the Schrdinger equation equation). The method provides very accurate coordinates of atoms in molecules and crystalline cells using the WIEN2K [20] program package.

Our analysis: After counting all the variables, we have reached for each coordinate number 7 . The basis has 6 variables, $\mathrm{R}(3,6)=18$, i.e., 11 other (additional) variables. It means -11 possible further properties. All of 
these cases show that the formulae and properties used in them represent a rather narrow way of learning the investigated systems.

Similarly, as in the next subchapter we ask: Where are these further (additional) possible variables?

- They are not used because it was not necessary for function of the formula, or they represent features that are not easy to interpret or are not measurable in our sense.

- The structure of the systems is hard to be expressed by mathematics derived from our arithmetic or the structure of system representation is not be attainable by our mathematics.

There are other circumstances:

- Other (additional) variables have low sensitivity in the relationships in which they emerge. Therefore, they are not stated.

- We cannot see other (additional) variables because they are included both in the definitions of the variables used and in the methods we measure these (used) variables.

- Additional variables do not express motion.

On the other hand, it is known from practice that in all these cases the formulae are "saturated" and provide that are accepted.

\section{Shapes - Approximations}

In many cases, no formulas are given (the solution is unattainable or unstable) and no more than patterns obtained from the models are reported. (Even neural models are approximations).

In the operations around the Schrödinger equation we find a number of approximations that simplify the computational demands on the solution (even today.) (The pure Schrödinger equation is suitable especially for ab initio calculations.)

One of the interesting approximations of the Schrödinger equation is Self Consistent Field approximation (SCF), [22]. In it, the variable - Hartree Potential for the $i$ th electron - is important:

$$
V_{H^{(i)}}=\int \rho\left(\mathbf{r}^{\prime}\right)\left(\left(\mathbf{r}-\mathbf{r}^{\prime}\right) /\left|\mathbf{r}-\mathbf{r}^{\prime}\right|^{3}\right) \mathrm{d} \mathbf{r}^{\prime},
$$

where $\rho(\mathbf{r})$ is the total electron density (after Fock's modification), $\mathbf{r}$ is the position vector of the $i$ th electron, $\mathbf{r}^{\prime}$ is the auxiliary variable position vector. (For various reasons, it is considered advantageous to express the position vector $\mathbf{r}$ in the form of the sum of two vectors $\mathbf{r}=\mathbf{R}_{\mathbf{I}}+\mathbf{r}^{\prime}$, where $\mathbf{R}_{\mathbf{I}}$ is a constant position vector of the nucleus "I" on which the atomic orbital is located and $\mathbf{r}^{\prime}$ is a new variable vector related to nucleus "I".

According to our analysis we find that we have 4 variables, 3 of which are bases, $\mathrm{R}(3,3)=6$, i.e., there are 2 other possible (additional) variables. In other words, the image of the system that is described by Hartree's potential can be improved by two other properties.

In [22] we read: the result of Hartree-Fock's approximation is the addition of the exchange term (which represents the contribution from the antisymmetry of the multi-electron wave function) to the overall potential. Another term that needs to be added to Wigner is the correlation energy that originates from the neglected electron-electron interaction. But these are two new independent variables (from at least three $-\mathrm{R}(3,4)=9$ ), which we add to the formula improve its function. Another case of systems that use approximations is so-called chaotic dynamic systems. They are typically characterized as systems that are extremely sensitive to setting initial conditions or setting parameters.

In the area of chaotic systems, we encounter cases for which we know formulae. Such are the systems of Lorenz, Roessler, Henon [6] and others. In these systems, we study the influence of the structure of the system and the setting of its parameters in relation to a chaos measurement function (e.g., correlation dimension, capacity dimension, fractal dimension, Lyapunov exponent, etc.) The second cases are systems for which we have no formulas and we only have data from system behavior measurements. These are cases of biosignals, ECG signals, EEGs and so on. Lorenz Equations have the following form:

$$
\begin{aligned}
\mathrm{d} x / \mathrm{d} t & =-\sigma(x-y), \\
\mathrm{d} y / \mathrm{d} t & =-x z+r x-y, \\
\mathrm{~d} z / \mathrm{d} t & =x y-b z,
\end{aligned}
$$

where $\sigma, b$, and $r$ are real parameters and are assumed to be positive. The basis has three elements $y=f(x, z, t)$ it means 6 elements in matroid and two other (additional) variables. Lorenz equations represent an attempt of their author to predict the weather. However not even a short-term prediction was successful. Here, other dimensions (independent variables) would fit. Until now, failed. To eliminate the chaos of the Lorenz system, you can use two additional variables that subtract nonlinear members.

$$
\begin{aligned}
& q_{1}=x z, \\
& q_{2}=-x y,
\end{aligned}
$$


But this is not any benefit for practice. If we wanted to improve the formulas, we would have to add 5 variables ( 2 additional and 3 new $-\mathrm{R}(3,4)=9$ ) to the list of variables, one of which could be independent. This is already a challenge.

There are many papers that have been published since 1996 in the area of HRV and arrhythmias research with regard to chaos (e.g., [17]). We introduce here our experience. In works [11] and [23], a MatLab / Simulink / Fuzzy toolbox model of cardio-vascular systems was developed and was tuned according to the values measured in patients. Here there are only brief conclusions:

- It was not possible to write an analytical solution for the HRV waveforms because the model was complicated and contained a number of fuzzy blocks with crossed delayed feedbacks.

- For the approximation of the HRV waveforms, the values of correlation and fractal dimensions were calculated. In both cases, the success of calculations was dependent on data quality.

- For Correlation Dimension (CD) it was the problem of saturation (reaching a stable horizontal phase) of curve $\mathrm{CD}=F\left(\operatorname{dim}_{e}\right),\left(\operatorname{dim}_{e}\right.$ - the dimension of embedding, $)[11]$.

- Neural network applications proved to be better than analyzing HRV approximations using nonlinear methods, [23]. (Approximation of approximations.)

The paper [12] studied the accuracy of meteorological data measured on the ecosystem of the Třeboň Basin (South Bohemia). The values of fractal data dimension were computed very low from 0.21 to 0.2699 - depending on places where the data originated. Considering that the FD Lorenz system is 2.09 [19] (CD is 2.07), it is a very regular meteorological data.

Note (3). In this moment is good to repeat the goal of this paper. Though we have been speaking now about the behavior of systems and what is encoded in approximations we concentrate attention on spaces of properties in which approximations are developed. From this point of view the relations and functions between variables represent secondary aspects.

As in the case of systems characterized by the formulae, as well as in the systems for which only approximation is known, there is a correlation between the matroid of the variables, the size of its base and the number of other possible variables. The more variables we know in approximations, the more other (additional) variables, the more variables we may to add to increase system dimensions and improve results.

\section{Qualitative Shapes}

From formulae through approximations, we come to shapes which have not been thoroughly measured and tested, but they have been designed with the help of an expert in the field and contain answers to questions like:

- Which variables can depend on system outputs?

- Which variables can describe system behavior?

For these descriptions, an inventory of effects cannot be taken as a matroid base and additional variables are not considered as in previous cases. Conversely, we will only get to the matroid with other operations.

If we compare this phase of shape development with formulae and approximations, it is more a development of proto models than a hard formalization and modeling. On the other hand, we admit that at this stage, all the formulae and approximations we mentioned above could have been. Such proto-models arise under the supervision of experts, and if a sophisticated information technology is chosen, they can be used very soon, not only after complete verification. (Sometimes, in particularly complex large and inaccessible systems, such verification is not possible.)

In addition to these rather intuitive notes on the development of qualitative models, there is also the extension of the list of classical intentions - "proposition", "quantities", "properties" and "individual offices", [3] by two "new"

$$
\begin{aligned}
\text { Specifiers }: S S P W & \longrightarrow \text { Specifications } \\
\text { Synthesisers }: S S P W & \longrightarrow \text { Operations, }
\end{aligned}
$$

where SSPW is a "Space of States of Possible Worlds" and Specifications and Operations are values of mappings Specifiers and Synthesisers. In analogy with disciplines developed above values of classical intentions (as for, e.g., quantities (variables) are numbers) is the discipline developed above values "Specifications" the "Theory of Interpretation" and the discipline developed above values "Operations" is "Synthetic and construction algebras".

These two intentions introduce a new style of work with qualitative shapes. We leave quantities and operations with them and we near to cognitive categories and operations with them. The most impressive is the Process of Interpretation (as one of principle concepts of Theory of Interpretation). There are a few types of processes of interpretation however the most important for us in this paper are two: 
- Interpretation for Identification (What it is? What does it mean?, etc.),

- Interpretation for Function (Which function has this object? For what we need this object?, etc.) The results of interpretation process are qualitative constructions (responses for questions, solutions of problems, etc.). These shapes are called in this paper qualitative shapes. We introduce here only two examples of qualitative shapes:

Qualitative Modeling and Monitoring of Selected Ecosystem Functions: In the article [5] has been described an ecological system using 19 representative states. State diagrams of transitions between the states were further processed into the Hasse diagram and the behavior of the system using rules (IF / THEN) with corresponding coefficients (Supp and Conf) was described. A matroid model was also built. Four 5 element model bases have been found. The bases represented subsets of states that most characterized the behavior of the system with respect to a certain problem factor " $\alpha$ ". The problem factor " $\alpha$ " was formulated as the following: "The stabilization of a sufficient humidity in the interaction zone $(0-200 \mathrm{~m})$ above the landscape surface of the ecosystem".

$$
\begin{aligned}
& \mathrm{B} 1=\{\mathrm{S} 2(+), \mathrm{S} 7(+), \mathrm{S} 12(+,-), \mathrm{S} 14(-), \mathrm{S} 15(+,-)\}, \\
& \mathrm{B} 2=\{\mathrm{S} 2(+), \mathrm{S} 7(+), 8(-), \mathrm{S} 12(+,-), \mathrm{S} 15(+,-)\}, \\
& \mathrm{B} 3=\{\mathrm{S} 4(+), \mathrm{S} 7(+), 12(+,-), \mathrm{S} 15(+,-), \mathrm{S} 19(-)\}, \\
& \mathrm{B} 4=\{\mathrm{S} 7(+), \mathrm{S} 10(+,-), 12(+,-), \mathrm{S} 14(-), \mathrm{S} 19(-)\} .
\end{aligned}
$$

Signs $(+,-)$ describe influence of the state in given problem factor " $\alpha$ ". States represented the following

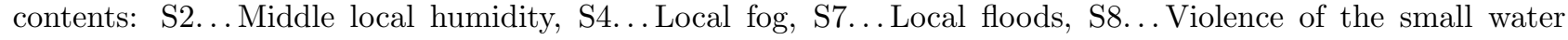
cycle, S10... Snow, S12. . Very cloudy weather and overcast, S14 . . Strong wind, S15... Storm, S19... Violated evapotranspiration.

Here we can see that qualitative shapes are formed in spaces that are rather open (compared to the spaces of the properties of formulae) and that the numbers of "variables" can exceed the cardinalities of matroids formed according to their bases $(\mathrm{R}(3,5)=14, \mathrm{R}(3,6)=18$.) (This was also investigated in article [6].)

Interpretation of States Structures in the Control of Development of Ecosystems: A similar use of matroid and its bases was published in [18]. But that was done by looking for a new unknown AXX factor that would influence climate change and was independent on the variables considered. His interpretation (as a construction of possibilities of what property it might be) proceeded in such a way that the formal result of the method was reflected by human solver, who then found a solution. (Emergent Solution.) The method as a whole was semi-formal. A situation where an explanation of the new state structure generated by some inference module is required is by the means of formal logic as well as by the means of the theory of problem solving impossible. The article proposes a method that allows to avoid this fundamental difficulty.

Note (4). After development of some basic concepts as "Interpretation Reference Context" we could turn back to Fig. 1 and try to achieve purposeful results by interpretation process.

\section{Conclusion}

We dealt with the issue of volumes and contents of spaces of properties in which is described the behavior of complex systems by means of shapes. We considered shapes as formulae, approximations and qualitative shapes. We have shown the relationship between matroids, their bases, and Ramsey numbers. When searching for answer to the question of why only the minimum number of possible variables is used in the formulae, we have come to the following factors:

- It depends on the definition of the variables used. Perhaps some variables aggregate more elementary variables without being able to observe them.

- Most examples have been taken from physics, where most of the results are verified by measurement. The volume of space of properties depends, therefore, on the methods used for measurement of the used properties.

- In formulae is usually used the mathematics based on a certain type of arithmetic. It is possible that other (additional) variables are not reachable by this type of arithmetic.

- Another reason why some variables are "invisible" is that they do not "express" themselves by motion. They could be detected as emergences.

The same qualities as for formulae have been followed for approximations. Approximations have been considered as:

- Simplifying relationships (e.g., Hartree Potential for simplifying the solution of Schrödinger equation).

- Characteristics given by means of correlation dimension, fractal dimension and Lyapunov exponent for chaotic dynamic systems. 
The last types of shapes were qualitative shapes.

- There has been mentioned one operation with qualitative shapes - Interpretation.

- The results of interpretation process are qualitative constructions (e.g., global solutions of problems without necessity of use of numerical structures).

Acknowledgement: This research has been supported by means of SGS17/P2301/OHK2-015. This support is very gratefully acknowledged.

\section{References}

[1] Kinsner, W.: Complexity and its measures in cognitive and other complex systems. In: 7th IEEE International Conference on Cognitive Informatics - ICCI 2008, 2 - 75. Stanford, CA, USA (2008)

[2] Stewart, I.: Nature's Numbers, The Unreal Reality of Mathematical Imagination. The Orion Publishing Group, Great Britain, (John Brockman, ed.) (1995)

[3] Tichy, P.: Foundations of Freges Logic. De Gruyter, Berlin (1988)

[4] Bila, J.: Emergent Phenomena in Complex Systems and their Detection. International Journal of Enhanced Research in Science Technology and Engineering 6(12), 40-53 (2017).

[5] Bila, J., Pokorny, J., Jura, J., Bukovsky, I.: Qualitative Modeling and Monitoring of Selected Ecosystem Functions. Ecological Modeling 222(19), 3640-3650 (2011).

[6] Bila, J.: The Syntheses of Technological Materials as Emergences in Complex Systems. In: R. Matousek (ed.) Proceedings of 20th International Conference on Soft Computing - MENDEL 2014, no. 20 in MENDEL, pp. 305-310. Brno University of Technology, VUT Press, Brno (2014)

[7] Din, H.U., Reshak, A.H., Bila, J., et. al.: Structural, elastic, thermal and electronic properties of $\mathrm{M}_{2} \mathrm{X}$ $(\mathrm{M}=\mathrm{Sr}, \mathrm{Ba}$ and $\mathrm{X}=\mathrm{Si}, \mathrm{Ge}, \mathrm{Sn})$ compounds in anti-fluorite structure: first principle calculation. Indian Journal of Physics 89(4), 369-375 (2015)

[8] Reshak, H.A., Khan, S.A., Kamarudin, H., Bila, J.: NaAuS chicken-wire-like semiconductor: Electronic structure and optical properties. Journal of Alloys and Compounds 582, 6-11 (2014)

[9] Sikander, A., Bila, J., Karmarudin, H., Reshak, A.H.: Electronic Structure, Electronic Charge Density and Optical Properties of 3-methyl-1,4-dioxo-1, 4-dohydronaphthalen-2-ylsulfanyl $\left(\mathrm{C}_{13} \mathrm{H}_{10} \mathrm{O}_{4} \mathrm{~S}\right)$. International Journal of Electrochemical Science 9, 445-459 (2014)

[10] Horak, J., Krlin, L.: Deterministic Chaos and mathematical models of turbulency. ( In Czech.) ACADEMIA, Prague (1996)

[11] Bila, J., Zitek, P., Kuchar, P., Bukovsky, I.: Heart Rate Variability: Modelling and Discussion. In: Int. IASTED Conf. - NN 2000, pp. 54-59, Pittsburgh, USA (2000)

[12] Jura, J., Novak, M.: Analysis of the hydrometeorological data using the Fractal dimension estimation. In: Proceedings of 21st International Conference on Process Control (PC), 2017, pp. 137-142. Strbske Pleso, Slovakia (2017)

[13] Oxley, J.G.: Matroid Theory, Reprinted Edition. Oxford Science Publications, Oxford (2001)

[14] Ramsey, F.P.: On a Problem of Formal Logic. Proc. London Math. Soc. 30, 264-286 (1930)

[15] Weinstein, W.: Ramsey Number. A Wolfram Web Resource. http:/mathword.wolfram.com/RamseyNumber.html (2004) [Online; accessed 17-May-2004]

[16] de Broglie, L.: La mecanique ondulatoire et la structure atomique de la matiere et du rayonnement. J. Phys. Radium 8, 225-241 (1927)

[17] Hagerman, I., Berglund, M., Lortin, M., Nowak, J., Sylven, Ch.: Chaos-related deterministic regulation of heart rate variability in time- and frequency domains: effects of autonomic blockade and exercise. Cardiovascular Research 31(3), 410-418 (1996)

[18] Bila, J., Novak, M.: Interpretation of States Structures in the Control of Development of Ecosystems. Journal of Engineering Research in Africa 18, 85-94 (2015)

[19] Budai, J.: Calculating Fractal Dimension of Attracting Sets of the Lorenz System. Dynamics at the Horsetooth 6, 1-12 (2014)

[20] Blaha, P. Schwarz, K., Luitz, J.: WIEN97. A full potential linearized augmented plane wave package for calculating crystal properties. Karlheinz Schwarz. Techn. Univesity at Wien, Austria (1991)

[21] Bla, J., Jura, J., Novk, M.: Application of Fuzzy Logic for Monitoring of Appearance of Heat Waves in Large Towns. Submitted for MENDEL 2018 (2018)

[22] Sahni, V.: Quantal Density Functional Theory. Springer Verlag, Heidelberg (2004)

[23] Bila, J., Ulicny, D.: Analysis of Chaotic Signals: Non-Linear Methods versus Neural Networks. In: Proceedings of International Carpathian Control Conference ICCC'2002, pp. 481-486 (2002) 